\title{
CORRECTION
}

\section{Correction: Ab initio study of anisotropic mechanical and electronic properties of strained carbon-nitride nanosheet with interlayer bonding}

\author{
Hao Cheng ${ }^{1,2}$, Jin-Cheng Zheng ${ }^{1,2, \dagger}$ \\ ${ }^{1}$ Department of Physics and the Collaborative Innovation Center for Optoelectronic Semiconductors and Efficient Devices, \\ Xiamen University, Xiamen 361005, China \\ ${ }^{2}$ Department of Physics, Xiamen University Malaysia, 439000, Sepang, Selangor, Malaysia \\ Corresponding author. E-mail: ${ }^{\dagger} j c z h e n g @ x m u . e d u . c n$
}

Correction to: Front. Phys. 16(4), 43505 (2021), https://doi.org/10.1007/s11467-021-1077-6

Unfortunately, the original article was published with wrong ESM files. The correct ESM files are replaced with the existing files.

*This article can also be found at http://journal.hep.com.cn/fop/EN/10.1007/s11467-021-1106-5. 


\title{
$A b$ initio study of anisotropic mechanical and electronic properties of strained carbon-nitride nanosheet with interlayer bonding
}

\author{
Hao Cheng ${ }^{1,2}$, Jin-Cheng Zheng ${ }^{1,2, \dagger}$ \\ ${ }^{1}$ Department of Physics and the Collaborative Innovation Center for Optoelectronic Semiconductors \\ and Efficient Devices, Xiamen University, Xiamen 361005, China \\ ${ }^{2}$ Department of Physics, Xiamen University Malaysia, 439000 Sepang, Selangor, Malaysia \\ Corresponding author. E-mail: ${ }^{\dagger} j c z h e n g @ x m u . e d u . c n$ \\ Received January 22, 2021; accepted April 27, 2021
}

\begin{abstract}
Due to the noticeable structural similarity and being neighborhood in periodic table of group-IV and $-\mathrm{V}$ elemental monolayers, whether the combination of group-IV and $-\mathrm{V}$ elements could have stable nanosheet structures with optimistic properties has attracted great research interest. In this work, we performed first-principles simulations to investigate the elastic, vibrational and electronic properties of the carbon nitride $(\mathrm{CN})$ nanosheet in the puckered honeycomb structure with covalent interlayer bonding. It has been demonstrated that the structural stability of $\mathrm{CN}$ nanosheet is essentially maintained by the strong interlayer $\boldsymbol{\sigma}$ bonding between adjacent carbon atoms in the opposite atomic layers. A negative Poisson's ratio in the out-of-plane direction under biaxial deformation, and the extreme in-plane stiffness of CN nanosheet, only slightly inferior to the monolayer graphene, are revealed. Moreover, the highly anisotropic mechanical and electronic response of CN nanosheet to tensile strain have been explored.
\end{abstract}

Keywords carbon-nitride, anisotropy, Poisson's ratio, strain engineering, in-plane strength, interlayer bonding

\section{Introduction}

The fascinating mechanical and electronic properties of monolayer graphene and its heterostructures, derived from its unique hexagonal symmetry with $s p^{2}$ intralayer bonding, such as high carrier mobility, massless Dirac fermions, and ultrastrength in-plane stiffness, have attracted significant research interest in two-dimensional (2D) materials for the last decade [1-3]. Not only have the extraordinary properties of monolayer graphene been revealed, but also the existence of other group-IV elements forming honeycomb lattices, i.e., silicene and germanene, has been demonstrated $[4,5]$. However, the electronic application of group-IV element monolayer is limited by the band gap closure around the Fermi level [6]. Recent synthesis of phosphorene has demonstrated the actual existence of group- $\mathrm{V}$ elemental monolayer and its intriguing properties, such as direct band gap, high carrier mobility, and prevalent optical properties have been revealed [79]. Besides, the theoretical predictions of stability, electronic structures $[10,11]$ and experimental synthesis [11]

\footnotetext{
* arXiv: 2104.14224. This article can also be found at http://journal.hep.com.cn/fop/EN/10.1007/s11467-0211077-6.
}

of group-III element monolayer have been systematically studied. Because of their noticeable structural similarity and being neighborhood in periodic table of groupIV and -V elemental monolayers, one would ask, whether the compounds consisting of group-IV and $-\mathrm{V}$ elements could have stable nanosheet structures with optimistic properties. Plentiful attempts have been directed towards synthesizing group IV-V compounds, e.g., carbon nitride $(\mathrm{CN})$ and carbon phosphide $(\mathrm{CP})$, from three-dimensional (3D) bulk phases to 2D nanosheets [12]. Some experiment results have manifested the existence of $\beta-\mathrm{C}_{3} \mathrm{~N}_{4}$ showing comparable hardness to diamond [13-15]. Moreover, the possibility of a wide range of other nitrogen concentration is shown in other experimental data $[16,17]$. The synthesis of amorphous CP films using radio frequency plasma deposition method has been reported by Pearce et al. [18]. Different C/P ratios can be achieved by adjusting the ratio of $\mathrm{PH} 3 / \mathrm{CH} 4$ gas during the synthesis. In other experiments, the pulsed laser deposition $[19,20]$ and magnetron sputtering techniques [21] were used in producing CP films.

Several possible crystal structures for $\mathrm{CN}$ bulk phase have been suggested via theoretical study, including the eight structures considered by Cote and Cohen, among which the GaSe layered phase (honeycomb crystal) is assumed to be the most energetically favorable struc- 
ture [22]. Besides that, in our earlier work [23], it is predicted that the GaSe layered phase are energetically favorable for all group IV-V compounds except for SnSb, and it shows semiconductor characters while other structures show metallic properties. For 2D nanosheets, Wang et al. suggested three kinds of potential structures for $\mathrm{CP}$ which they calculated super carrier mobility and strong anisotropy [24]. On the other hand, the electronic and thermal properties of multi-layer nanosheets and its heterostructures are substantially affected by the interlayer interaction [25-29]. There are generally two ways for layer interactions, van der Walls (vdW) force and covalent bonding. The covalent bonding naturally brings in stronger cross-plane coupling than the weak vdW force. Recent experiments have shown that the $\boldsymbol{\sigma}$ bonding of $\mathrm{C}$ atoms between the layers of graphite could be induced by visible-light irradiation [30]. The cooperative and nonlinear formation mechanisms of this excited interlayer $\boldsymbol{\sigma}$ bonds are demonstrated [31]. The influence of these interlayer interaction to the thermal properties of multi-layer graphene has been conducted both experimentally and theoretically [32-34].

In this paper, we performed first-principles simulations to investigate the elastic, vibrational and electronic properties of the $\mathrm{CN}$ nanosheet in the puckered honeycomb structure with covalent interlayer bonding. It has been demonstrated that the structure stability of $\mathrm{CN}$ nanosheets is essentially maintained by the strong interlayer $\boldsymbol{\sigma}$ bonding between two adjacent carbon atoms in the opposite atomic layers. Our numerical results show a negative Poisson's ratio in the out-of-plane direction under biaxial deformation. We attribute this abnormal Poisson's ratio to the introduction of additional degrees of out-of-plane atomic relaxations by the formation of covalent interlayer bonding in $\mathrm{CN}$ nanosheet. With the application of various uniaxial and biaxial tension strains, the highly anisotropic mechanical and electronic response of $\mathrm{CN}$ nanosheet to the the strain has been explored. It is identified that the tensile strength of $\mathrm{CN}$ nanosheets are dictated by the instability of out-of-plane transverse phonon branch ZA for armchair tension $\epsilon_{x x}$, but in-plane transverse phonon branch TA for the zigzag tension $\epsilon_{y y}$. Our calculations indicate that the indirect band gap of CN nanosheet could be significantly tailored through strain engineering which manifests its potential application in optoelectronic fields.

\section{Method}

We carried out the first-principles calculations based on the density functional theory (DFT) as implemented in the Quantum Espresso simulation package [35]. The Perdew-Burke-Ernzerhof (PBE) exchangecorrelation functional [36] along with the projector augmented wave (PAW) pseudopotentials [37] were adopted for the self-consistent total energy and electronic-structure calculations. The kinetic energy cutoff for wave functions and charge density were set to be 85 Ry and 595 Ry respectively. To model $\mathrm{CN}$ nanosheets, a four-atom primitive cell containing two carbon atoms and two nitrogen atoms was chosen with periodic boundary condition applied (see Fig. 1). Specifically, for stress-strain calculations, an eight-atom orthogonal unit cell was employed to achieve uniaxial stress along the armchair and zigzag direction (Fig. 1, dashed boxes). A Monkhorst-Pack grid of $(25 \times 25 \times 1) k$-points was used to sample the first reciprocal Brillouin Zone (BZ). The energy convergence criteria for electronic and ionic iterations were set to be $10^{-10} \mathrm{Ry}$ and $10^{-6} \mathrm{Ry}$, respectively. All atomic positions and unit cell were optimized until the atomic forces were less than $10^{-5} \mathrm{Ry} / \mathrm{au}$. A minimum of $20 \AA$ vacuum spacing in the out-of-plane direction was applied to prevent interaction from adjacent supercells. The phonon disper-
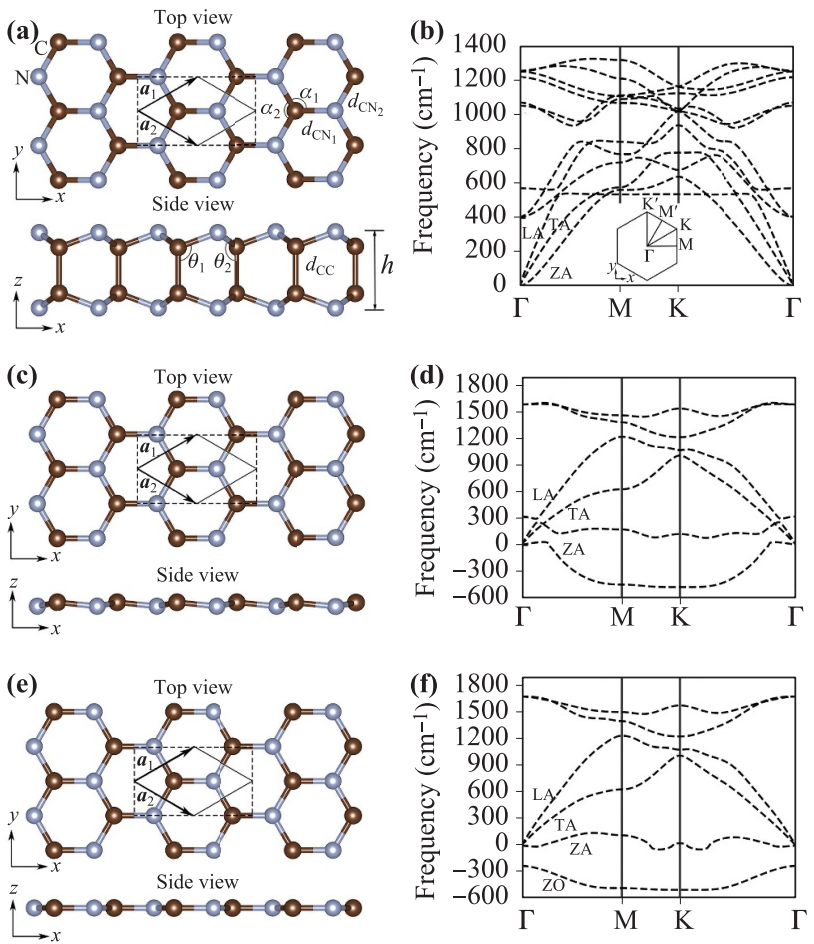

Fig. 1 The crystal structures including top and side views of CN nanosheets forming (a) LB geometry with covalent interlayer bonding $d_{\mathrm{CC}}$, (c) LB geometry without interlayer bonding (monolayer), (e) PL geometry (monolayer). The dark brown and light blue spheres are $\mathrm{C}$ and $\mathrm{N}$ atoms, respectively. The Bravais lattice vectors of primitive are indicated by $\boldsymbol{a}_{1}$, $\boldsymbol{a}_{2}$ and vector directions are represented with arrows. The $x$ and $y$ directions correspond to the armchair and zigzag directions, respectively. An eight-atom unit cell for uniaxial tension calculation is denoted with dashed box. The corresponding ground state phonon dispersion curves of these three atomic configurations are presented in (b), (d), and (f). The acoustic phonon and imaginary phonon are indicated. The unstable phonon branch is ZA for (c), ZO and ZA for (e). Inset shows the deformed first BZ under uniaxial tension. 
sion curves were calculated by diagonalizing the dynamical matrix based on the density functional perturbation theory.

It is well known that the PBE functional may underestimate the calculated band gaps. In this work, the frequency-dependent $G W$ quasiparticle calculations were performed to correct the ground state band gaps obtained by PBE. Furthermore, a scissors operator method has been implemented to correct band gaps under tensile strains $[38,39]$. The $G W$ calculations were conducted as a "one-shot" correction to self-consistent PBE calculations. This approach, denoted as $G_{0} W_{0}$, has yielded remarkably accurate band structures for many materials [40-42]. Various tests were carried out to achieve convergence regarding $k$-points, vacuum level, number of bands, and kinetic energy cutoff. Final $G_{0} W_{0}$ corrections on the PBE bands gaps were obtained by using $(17 \times 17 \times 1) k$-points in $\mathrm{BZ}$, $25 \AA$ vacuum spacing, 120 bands and default cut-off potential for $G_{0} W_{0}$.

In contrast to the flatness of graphene, the $\mathrm{CN}$ nanosheet we proposed here is a puckered honeycomb structure with covalent interlayer bonding. The bravais lattice vectors of primitive cell are defined as $\boldsymbol{a}_{\mathbf{1}}$ and $\boldsymbol{a}_{\mathbf{2}}$, shown in Fig. 1(a). The armchair and zigzag directions are along the $x$ and $y$ directions, respectively. Under uniaxial tension, the original lattice symmetry is broken, thus the structure parameters along armchair direction and zigzag direction are different (the subscripts are assigned to distinguish them). The intralayer bonding $d_{\mathrm{CN}_{1}}$ and $d_{\mathrm{CN}_{2}}$ are formed between carbon and nitrogen atoms in the same atomic layer, while the interlayer bonding $d_{\mathrm{CC}}$ is formed between two adjacent carbon atoms in the opposite atomic layers. The in-plane $\mathrm{N}-\mathrm{C}-\mathrm{N}$ bond angles is named as $\alpha_{1}$ and $\alpha_{2}$, and out-of-plane $\mathrm{N}-\mathrm{C}-\mathrm{C}$ bond angle is named as
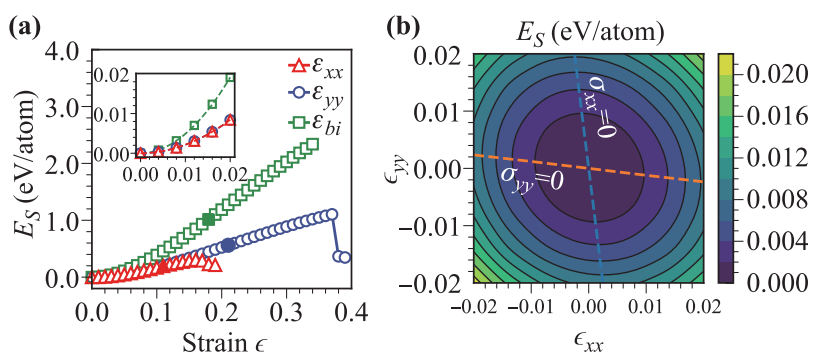

Fig. 2 (a) The strain energy $E_{S}$ of $\mathrm{CN}$ nanosheet as functions of tensile strain $\epsilon_{x x}, \epsilon_{y y}$, and $\epsilon_{b i}$ in uniform deformation region. The solid triangles, circles and squares indicate the condition where the peak stress could be attained along armchair, zigzag and biaxial tensions, respectively. Inset shows the polynomial regression of the initial strain curves for obtaining the corresponding elastic moduli. (b) The contour plot of strain energy $E_{S}$ with respect to the $\left(\epsilon_{x x}, \epsilon_{y y}\right)$ in the harmonic region. The energy-strain relation is fitted as quadratic polynomial $E_{S}=a_{1} \epsilon_{x x}^{2}+b_{1} \epsilon_{y y}^{2}+c_{1} \epsilon_{x x} \epsilon_{y y}$ with $a_{1}=b_{1}=14360.1 \mathrm{eV}$, and $c_{1}=3583.7 \mathrm{eV}$. The dashed lines denoted the uniaxial deformations along $x$ and $y$ directions, respectively. $\theta_{1}$ and $\theta_{2}$, respectively. The layer height of $\mathrm{CN}$ nanosheet is characterized by the atomic distance between two nitrogen atoms sited opposite in the out-of-plane direction, i.e., $h$. The first $\mathrm{BZ}$ is deformed under the uniaxial tension with high symmetry points splitting up, as shown in Fig. 1(b).

For the stress-strain relations, in order to make a direct comparison of 2D nanosheets with experiments and other theoretical results, we need to rescale the supercell stress by $\eta=Z / d_{0}$ to obtain the equivalent stress, where $Z$ is the cell length in the direction perpendicular to atomic layers and $d_{0}$ is the effective thickness of nanosheet. In this work, we take $d_{0}=5.69 \AA$ for $\mathrm{CN}$ nanosheet which is the equilibrium interlayer spacing of $\mathrm{CN}$ bulk phase calculated in previous work by Cohen et al. [22]. It is worthwhile to mention that the $\mathrm{vdW}$ interactions between adjacent atomic layers of $\mathrm{CN}$ nanosheet are neglected in our calculations because those vdW corrections are assumed to be much weaker than the covalent interlayer bonding $d_{\mathrm{CC}}$ of CN nanosheet [25].

\section{Results}

We first optimize the equilibrium structural parameters of $\mathrm{CN}$ nanosheets in 2D honeycomb lattices. In present work, we consider three atomic configurations in accordance with the rate of buckling in the out-of-plane direction, i.e., low-buckled (LB) (or puckered) geometry with covalent interlayer bonding, LB geometry without interlayer bonding, and planar (PL) geometry. The structural stabilities of these three atomic configurations are examined by calculating the phonon dispersion curves.

Figure 1 shows the optimized structure models along with the ground state phonon dispersion curves of these three atomic configurations of $\mathrm{CN}$ nanosheets. While the LB geometry without interlayer bonding and PL geometry both show imaginary (negative) frequencies in the phonon dispersion, the structural stability is exclusively attained by the LB geometry with covalent interlayer bonding $d_{\mathrm{CC}}$. Compared to the planar geometry, the out-of-plane transverse phonon branch $\mathrm{ZO}$ is stabilized through the buckling of $\mathrm{C}-\mathrm{N}$ atomic layers in the LB geometry [Figs. 1(d) and (f)]. The planar $s p^{2}$ orbital is slightly dehybridized with perpendicular $p_{z}$ orbital to form a $s p^{3}$-like orbital as a result of the buckling. This buckled stabilization mechanism could be interpreted as the in-plane $\boldsymbol{\pi}$ bonding formed between $\mathrm{C}$ and $\mathrm{N}$ atoms is not strong enough to maintain the planar geometry by comparison with the $\mathrm{C}-\mathrm{C}$ bonding in the monolayer graphene [43]. However, it is still unstable with respect to the out-of-plane transverse phonon branch $\mathrm{ZA}$ in the LB geometry without interlayer bonding. Through the coupling of two symmetrically buckled atomic layers in hexagonal lattice, as the formation of covalent interlayer $\boldsymbol{\sigma}$ bonds between two adjacent $\mathrm{C}$ atoms, the $\mathrm{CN}$ nanosheets is stabilized with 
Table 1 The calculated structure parameters of CN nanosheet. The value of angle between intralayer bond $\mathrm{C}-\mathrm{N}-\mathrm{C}$ and interlayer bond $\mathrm{N}-\mathrm{C}-\mathrm{C}, \alpha$ and $\theta ; \mathrm{C}-\mathrm{N}$ and $\mathrm{C}-\mathrm{C}$ bond length, $d_{C N}$ and $d_{\mathrm{CC}}$; lattice constant, $a$; layer height, $h$; and cohesive energy, $E_{\text {coh }}$ are given.

\begin{tabular}{cccccccc}
\hline Structure & $\begin{array}{c}\alpha \\
\left({ }^{\circ}\right)\end{array}$ & $\begin{array}{c}\alpha \\
\left({ }^{\circ}\right)\end{array}$ & $\begin{array}{c}d_{\mathrm{CN}} \\
(\AA)\end{array}$ & $\begin{array}{c}d_{\mathrm{CC}} \\
(\AA)\end{array}$ & $\begin{array}{c}a \\
(\AA)\end{array}$ & $\begin{array}{c}h \\
(\AA)\end{array}$ & $\begin{array}{c}E_{\text {coh }} \\
(\mathrm{eV} / \text { atom })\end{array}$ \\
\hline \hline $\mathrm{CN}$ & 110.3 & 108.6 & 1.46 & 1.63 & 2.38 & 2.65 & 8.36 \\
\hline
\end{tabular}

fourfold bonding of $\mathrm{C}$ atoms and threefold bonding of $\mathrm{N}$ atoms [Figs. 1(b) and (d)]. The coordination number of $\mathrm{C}$ and $\mathrm{N}$ atoms are consistent with the number of valence electrons needed to fully fill their electronic shell respectively. In contrast to the weak vdW interactions between few layers planar graphene, this strong interlayer $\boldsymbol{\sigma}$ bonding between $\mathrm{C}$ atoms together with the buckled $\mathrm{C}-\mathrm{N}$ atomic layers maintain the structure stability of $\mathrm{CN}$ nanosheet. Besides that, it is further confirmed by the comparison of the calculated cohesive energy among these three atomic configurations. The cohesive energy is defined as $E_{c o h}=\left[E_{t}(\mathrm{C})+E_{t}(\mathrm{~N})-E_{t}(\mathrm{CN})\right] / 2$, where $E_{t}(\mathrm{CN})$ is the total energy per pair atoms of optimized $\mathrm{CN}$ nanosheet; $E_{t}(\mathrm{C})$ and $E_{t}(\mathrm{~N})$ are the total energies of isolated $\mathrm{C}$ and $\mathrm{N}$ atoms corresponding to nonmagnetic state. The cohesive energy of LB geometry with covalent interlayer bonding, $E_{c o h}=8.26 \mathrm{eV} /$ atom, turns out to be much larger than other two atomic configurations without interlayer bonding (Table S1, supplementary material). Consequently, from the indication of structure stability analysis above, our theoretical results below will be built based on the LB geometry with covalent interlayer bonding $d_{\mathrm{CC}}$.

Table 1 summarizes the calculated equilibrium structural parameters of $\mathrm{CN}$ nanosheet. It is interesting to compare the lattice parameters of $\mathrm{CN}$ nanosheet with those of graphene and other hexagonal lattice structures. The bond length of intralayer $\mathrm{C}-\mathrm{N}$ bond of $\mathrm{CN}$ nanosheet, $d_{\mathrm{CN}}=1.46 \AA$, is approaching that of the $\mathrm{C}-\mathrm{C}$ bond in monolayer graphene $(1.44 \AA)$ and $\mathrm{B}-\mathrm{N}$ bond in boron nitride $(1.45 \AA)$, both having very high in-plane strength, but much shorter than the Mo-S bond in $\mathrm{MoS}_{2}(2.42 \AA)$ and all other potential hexagonal structure of binary monolayer of group-IV elements and group III-V compounds reported by Şahain et al. [43]. As for the interlayer $\mathrm{C}-\mathrm{C}$ bond, $d_{\mathrm{CC}}=1.63 \AA$, it is a bit longer than the bond length of $s p^{3}$ hybridized orbital in diamond (1.53 $\AA$ ). In addition, the in-plane bond angle $\alpha$ and out-of-plane bond angle $\theta$ are $108.6^{\circ}$ and $110.3^{\circ}$ respectively, which are quite close to that of $109.5^{\circ}$ in diamond. Briefly, the strong interlayer $\boldsymbol{\sigma}$ bonding together with the hybridized $s p^{3}$-like orbital underlie unusual in-plane and out-of-plane mechanical strength of $\mathrm{CN}$ nanosheet compared to other traditional 2D materials.

To further explore the impact of strain on vibrational, mechanical and electronic properties of $\mathrm{CN}$ nanosheet, we
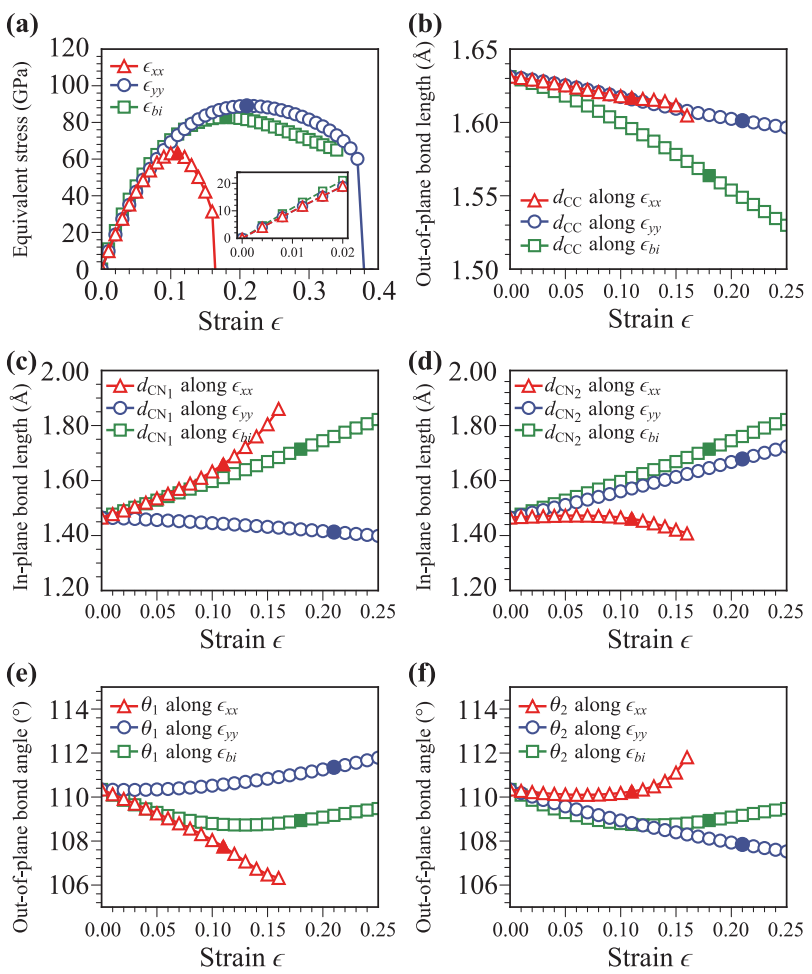

Fig. 3 (a) Calculated equivalent stress $\left(d_{0}=5.69 \AA\right)$ versus tensile strain $\epsilon_{x x}, \epsilon_{y y}$ and $\epsilon_{b i}$. (b) The variation of the outof-plane bond length $d_{\mathrm{CC}}$ with respect to the applied tensile strain. The in-plane bond length (c) $d_{\mathrm{CN}_{1}}$ and (d) $d_{\mathrm{CN}_{2}}$ as functions of the applied tensile strain. The out-of-plane bond angle (e) $\theta_{1}$ and (f) $\theta_{2}$ as functions of the applied tensile strain. The solid triangles, circles and squares indicate the condition where the peak stress could be attained along armchair, zigzag and biaxial tensions, respectively.

then stretch the eight-atom unit cell of $\mathrm{CN}$ with a series of incremental tensile strains including uniaxial tension along $x$ (armchair) direction $\epsilon_{x x}$, uniaxial tension along $y$ (zigzag) direction $\epsilon_{y y}$, and biaxial tension $\epsilon_{b i}\left(\epsilon_{x x}=\epsilon_{y y}\right)$. The atomic positions for each step are taken from the relaxed coordinates of the former step to ensure the continuity of strain path. For the uniaxial tension, the optimized structures are attained by simultaneously relaxing the strain components orthogonal to the applied strain. The variation in strain energies, $E_{S}$, by subtracting the total energy per atom of the deformed structure from the equilibrium one, is obtained as functions of elastic strain in both harmonic and anharmonic domain. Owing to the hexagonal symmetry of honeycomb structure, $\mathrm{CN}$ nanosheet shows isotropic strain energy response at small strains [inset in Fig. 2(a)]. In Fig. 2(b), we show the contour plot of strain energy as functions of strains $\left(\epsilon_{x x}, \epsilon_{y y}\right)$. The energy-strain relation is then obtained as a quadratic polynomial $E_{S}=a_{1} \epsilon_{x x}^{2}+b_{1} \epsilon_{y y}^{2}+c_{1} \epsilon_{x x} \epsilon_{y y}$, where $a_{1}, b_{1}$, and $c_{1}$ are parameters to be fitted. With the small strain limit, we set $a_{1}=b_{1}$ as $\mathrm{CN}$ nanosheet is elastically isotropic within the plane. The elastic stiffness 

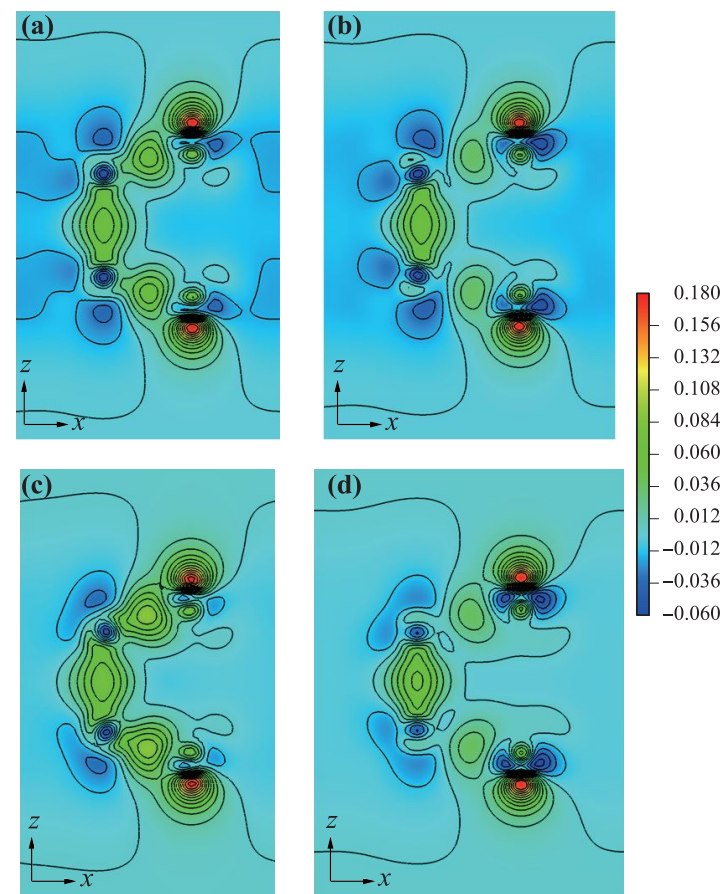

Fig. 4 Side view of the deformation charge density of $\mathrm{CN}$ nanosheet at (a) equilibrium state, (b) uniaxial tension $\epsilon_{x x}=$ 0.11 , (c) uniaxial tension $\epsilon_{y y}=0.21$, and (d) biaxial tension $\epsilon_{b i}=0.18$ where peak stress could be obtained. The blue and red colored isosurfaces represent the depletion and accumulation of charge, respectively. The contour interval is set to be 0.015 electron $/$ Bohr $^{3}$.

constants can then be expressed in terms of these fitting parameters, i.e., $C_{11}=2 a_{1} /\left(h \cdot A_{0}\right) ; C_{12}=c_{1} /\left(h \cdot A_{0}\right)$. Hence, the effective Young's modulus can be obtained as, $E=C_{11} \cdot\left[1-\left(C_{12} / C_{11}\right)^{2}\right]=\left[2 a_{1}-c_{1}^{2} /(2 a)\right] /\left(h \cdot A_{0}\right)$, where $A_{0}$ are the equilibrium surface area of the system. The $\mathrm{CN}$ nanosheet is calculated to have an effective Young's modulus $E=1013 \mathrm{GPa}$ which is comparable to that of $1 \mathrm{TPa}$ in monolayer graphene [44, 45].

Furthermore, we calculate the ideal strength of $\mathrm{CN}$ nanosheet under tensile strain. Figure $3(\mathrm{a})$ shows the equivalent stress as functions of tensile strains for $\mathrm{CN}$ nanosheet. Similar to the strain energy response, CN nanosheet has isotropic in-plane elastic response at small strains. There is no observable difference of the elastic response between the uniaxial tension $\epsilon_{x x}$ and $\epsilon_{y y}$ when strains are less than 0.02. While, at large strains, due to the broken of lattice symmetry, the elastic response in armchair direction becomes distinct from zigzag direction. The maximum stress for uniaxial tension along armchair direction is $\sigma_{x x}=63 \mathrm{GPa}$, at $\epsilon_{x x}=0.11$. Compared to that, $\mathrm{CN}$ nanosheet is stronger in the zigzag direction with maximum stress $\sigma_{y y}=89 \mathrm{GPa}$, at $\epsilon_{y y}=0.21$. Thus, we predict that the zigzag direction is $41 \%$ stronger than the armchair direction and can sustain $91 \%$ more strain. For the biaxial tension, the maximum stress is $82 \mathrm{GPa}$, at $\epsilon_{b i}=0.18$, which is between that along the armchair and zigzag tensions. It is worth mentioning that the ideal strength of CN nanosheet in armchair and zigzag direction are both smaller than those of $110 \mathrm{GPa}$ and $121 \mathrm{GPa}$ in monolayer graphene [46], but approaching that of $88 \mathrm{GPa}$ and $102 \mathrm{GPa}$ in single-layer hexagonal BN [47]. Moreover, we have explored the responses of the atomic relaxations, i.e., bond length and bond angle, with respect to the tensile strains, shown in Figs. 3(b)-(f). Most structure parameters show similar trend of variation (linear and monotonic) with respect to the tensions, especially within small strains. However, it is noted that the out-of-plane bond angle $\theta_{1}$ shows non-monotonic response (negative to positive slop ratio) with respect to the biaxial tension. This unique mechanical response indicates that there is competition between in-plane and out-of-plane atomic relaxation under tensile strain. The plot of deformation charge density gives a more vivid understanding of the variations of structure versus the tensile strains, as shown in Fig. 4. In the analysis of Poisson's ratio, we will give further explanation about the competition mechanism of this unique mechanical response.

The finite-deformation Poisson's ratio is defined as the ratio of the transverse strain to the applied strain [43, 46]. The in-plane Poisson's ratio is thus well defined as $v_{\|, x x}=$ $-\epsilon_{y y}^{\prime} / \epsilon_{x x}$ and $v_{\|, y y}=-\epsilon_{x x}^{\prime} / \epsilon_{y y}$ where the prime symbol denotes the relaxed strain. Nevertheless, for the out-ofplane Poisson's ratio, it depends on the determination of the deformation in the out-of-plane direction, i.e., $\epsilon_{z z}^{\prime}$. In our calculations, we make it equal the variation of layer height, namely, $\epsilon_{z z}^{\prime}=\left(h^{\prime}-h\right) / h$. Hence we obtain the out-of-plane Poisson's ratio $v_{\perp, x x}=-\epsilon_{z z}^{\prime} / \epsilon_{x x}$, $v_{\perp, y y}=-\epsilon_{z z}^{\prime} / \epsilon_{y y}$, and $v_{\perp, b i}=-\epsilon_{z z}^{\prime} / \epsilon_{b i}$. In Fig. 5(a), the in-plane Poisson's ratio $v_{\|, x x}$ and $v_{\|, y y}$ exhibit quantitatively different value in response to the applied strain. The variation of $v_{\|, x x}$ to the strain is much intense than that of the $v_{\|, y y}$. Despite the anisotropy of in-plane Poisson's ratio, it shows similar trend for $v_{\|, x x}$ and $v_{\|, y y}$ under applied strain (non-monotonic, negative to positive slop ratio). In contrast, the in-plane Poisson's ratio of monolayer graphene is a monotonically decreasing function of the tensile strain [46]. In Fig. 5(b), a negative Poisson's ratio $v_{\perp, b i}$ in the out-of-plane direction exists under biaxial tension at $\epsilon_{b i}=0.15$. In order to manifest the existence of this negative out-of-plane Poisson's ratio, Fig. 5(c) depicts the relaxation of layer height $h$ for same set of tensile strain. The layer height $h$ has the trend from decreasing to increasing with respect to the increase of biaxial tension. We attribute this negative out-of-plane Poisson's ratio to the additional degrees of out-of-plane atomic relaxation introduced by the formation of interlayer bonding of $\mathrm{CN}$ nanosheet. Unlike monolayer graphene, which is a truly 2D material with single atomic layer, the $\mathrm{CN}$ nanosheet is composed of four atomic layers $\mathrm{N}-\mathrm{C}-\mathrm{C}-\mathrm{N}$ with covalent interlayer bonding, or two buckling $\mathrm{C}-\mathrm{N}$ layers. As a consequence, the out-of-plane atomic relaxation, which is absent in monolayer graphene, will be involved in the 

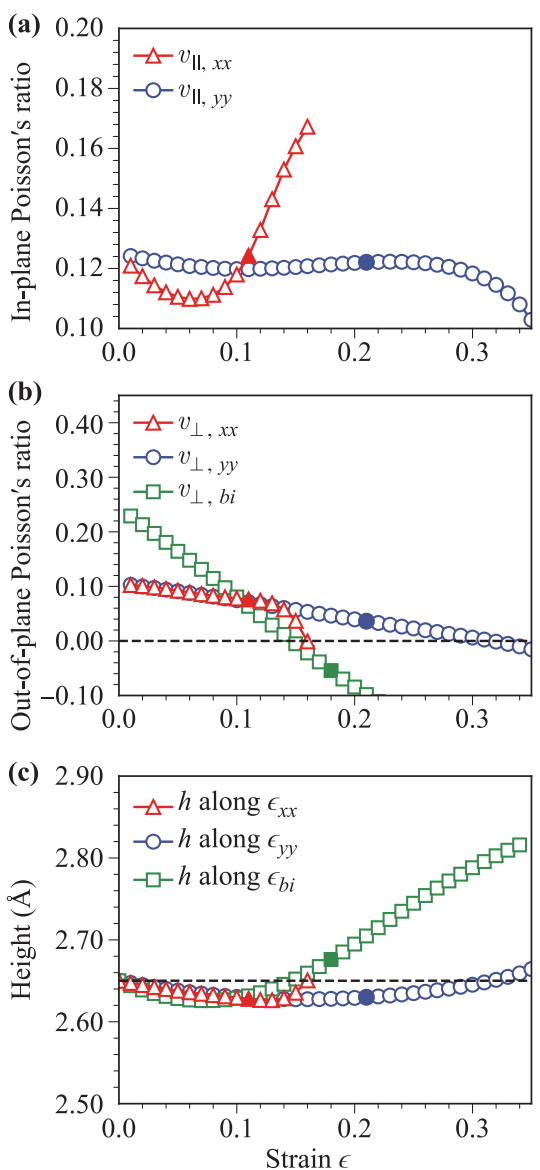

Fig. 5 Calculated (a) in-plane and (b) out-of-plane Poisson's ratio as functions of tensile strain. (c) The layer height relaxations are shown as functions of tensile strain. The solid triangles, circles and squares indicate the condition where the peak stress could be attained along armchair, zigzag and biaxial tensions, respectively.

mechanical response of $\mathrm{CN}$ nanosheet. The in-plane interactions thus competing with the out-of-plane covalent bonding make a complex atomic relaxation process under tensile strain. We note that the different definition on the Poisson's ratio are proposed in some other reports. The Poisson's ratio is defined as the partial derivative of transverse strain to axial strain, namely, $v=-\partial \epsilon_{\text {trans }} / \partial \epsilon_{\text {axial }}$, in Refs. [48, 49]. With this definition, there is still a negative Poisson's ratio $v_{\perp, b i}$ under biaxial tension beyond $\epsilon_{b i}=0.08$ in the CN nanosheet.

While the stress strain relation provides a rough indication of the ideal strength of CN nanosheet, it is still necessary to check whether the structure stability could be maintained before approaching the maximum stress, because the elastic instability may disrupt the homogeneous lattice structure on the strain path (the instability is not soft mode) [50, 51]. In Fig. 6, we present the anisotropic phonon dispersion curves for $\mathrm{CN}$ nanosheet under tensile strain. At uniaxial strain $\epsilon_{x x}=0.12$, there is no indication of phonon instability, that is, all of phonon frequen- cies shown are positive [Fig. 6(a)]. With the increasing of tension, the phonon instability does occur at $\epsilon_{x x}=0.13$ [Fig. 6(b)]. The eigenvectors of the unstable phonon indicate that this phonon branch is the ZA mode (Fig. S1, supplementary material). Noted that the critical strain $\epsilon_{x x}=0.13$ is beyond maximum strain $\epsilon_{x x}=0.11$ which means the ideal strength, $\sigma_{x x}^{i}=\sigma_{x x}^{m}=63 \mathrm{GPa}$, could be attained along armchair direction. In comparison, the phonon dispersion curves maintain stable until the uniaxial strain $\epsilon_{y y}=0.17$, but then initially has a negative frequency on the TA phonon branch at $\epsilon_{y y}=0.18$. The ideal strength is thus obtained ahead of the maximum strength, i.e., $\sigma_{y y}^{i}=87 \mathrm{GPa}$, at $\epsilon_{y y}=0.17$. For biaxial tension, the TA branch and TO branch phonon instability simultaneously occur at $\epsilon_{b i}=0.17$. It indicates that the failure mechanism under biaxial tension is the combination of the phonon instability and elastic instability.

Not only mechanical properties and atomic configuration but also the electronic properties of CN nanosheet, specifically its band gap, can be tailored through stretching. Our results on the electronic band structures of CN nanosheet and its variation with respect to different tensile strains are presented in Fig. 7. As expected, CN nanosheet
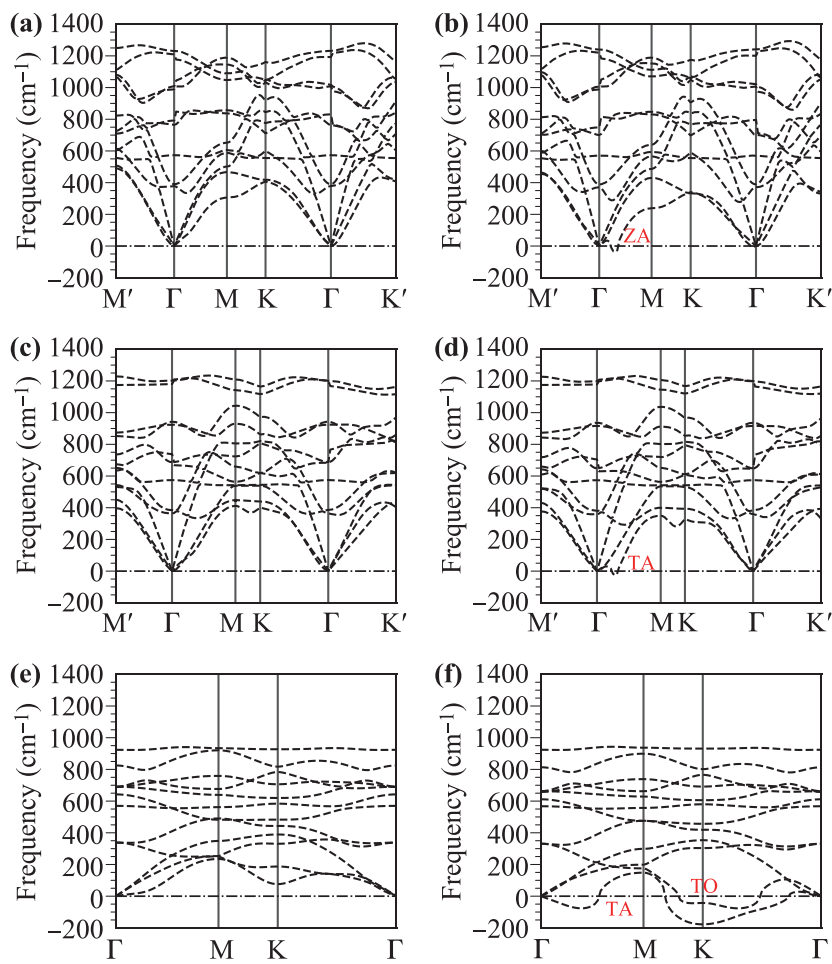

Fig. 6 Calculated vibration frequency versus $\mathbf{k}$ along the symmetry points in the first BZ under uniaxial tension (a) $\epsilon_{x x}=0.12$ and (b) $\epsilon_{x x}=0.13$; uniaxial tension (c) $\epsilon_{y y}=0.17$ and (d) $\epsilon_{y y}=0.18$; and biaxial tension (e) $\epsilon_{b i}=0.16$ and (f) $\epsilon_{b i}=0.17$. The unstable phonon mode is identified to be the out-of-plane transverse phonon branch ZA for (b) armchair tension and in-plane transverse phonon branch TA for (d) zigzag tension. 

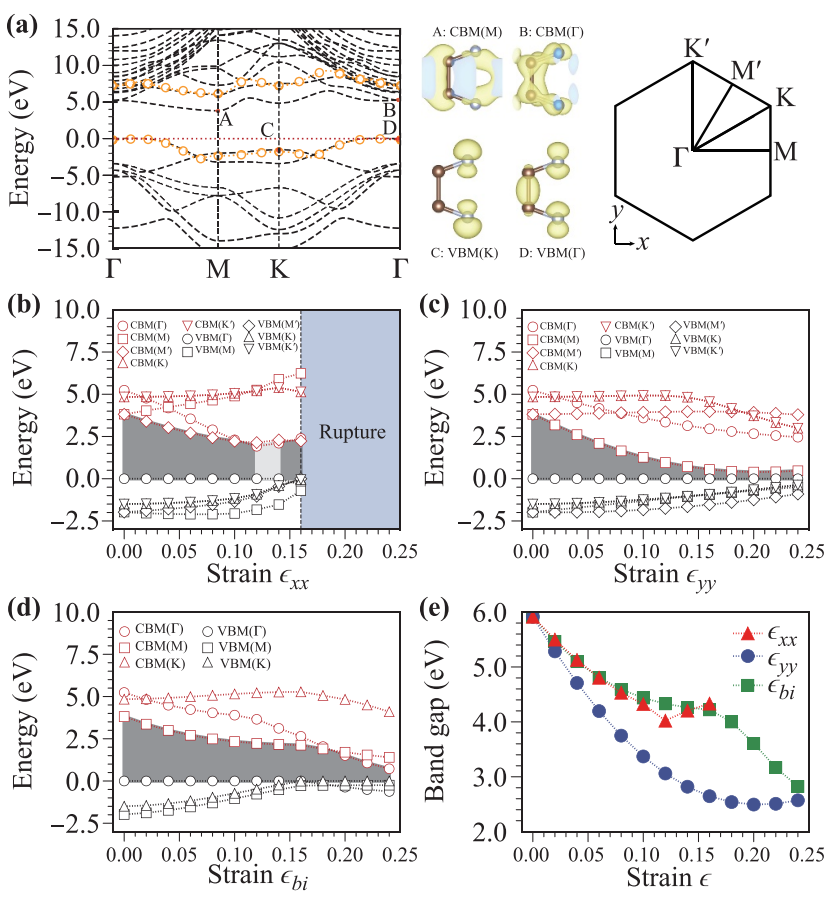

Fig. 7 (a) Calculated electronic band structure of $\mathrm{CN}$ nanosheet with PBE. Corrections on band gaps using $G_{0} W_{0}$ are indicated by yellow circles. The band decomposed isosurface charge densities for lowest two conduction bands and highest two valence bands are schematically described. The yellow and blue color represent the charge density and its cross section with periodic boundary, respectively. The deformed first BZ under uniaxial tension is also given. The variations of VBM and CBM obtained with PBE under tensile strain (b) $\epsilon_{x x}$, (c) $\epsilon_{y y}$ and (d) $\epsilon_{b i}$ are shown. The rupture along armchair tension is indicated. Dark (light) gray regions correspond to indirect (direct) band gaps. (e) Variations of band gap with respect to the applied strain is summarized. Results obtained at the PBE level and then corrected using the scissors operator method based on the $2.1 \mathrm{eV}$ difference between PBE and PBE $+G_{0} W_{0}$ band gap at the $\Gamma$ point. Noted that in panel (a) the Fermi energy is set to be zero. In panels (b), (c) and (d), the energies are referenced to the VBM to illustrate the modification of band gap.

with covalent interlayer bonding is determined to be semiconductor with an indirect band gap at equilibrium state. It is the fact that all electrons are involved in the formation of covalent bonds in both in-plane and out-of-plane direction. PBE predicts an indirect band gap of $3.8 \mathrm{eV}$ formed from the valance band maximum (VBM) at the $\Gamma$ point to the conduction band minimum $(\mathrm{CBM})$ at the $\mathrm{M}$ point and a direct band gap of $5.2 \mathrm{eV}$ at the $\Gamma$ point. With $G_{0} W_{0}$ corrections, the bands are shifted significantly in energy and the shift is not constant for different bands, resulting in a large increase of band gap at the $\mathrm{M}$ point than the $\Gamma$ point. The band gap corrections at high symmetry points are calculated to be $2.1 \mathrm{eV}$ at $\Gamma$ point, and $2.6 \mathrm{eV}$ at $\mathrm{M}$ and $\mathrm{K}$ point. This yields $6.2 \mathrm{eV}$ and $7.3 \mathrm{eV}$ for the indirect and direct transition, respectively. In contrast, regardless of the instability in the out-of-plane direction, the monolayer structures without the interlayer bonding [Figs. 1(c) and (e)] are calculated to display a metallic character (Fig. S2, supplementary material). With this in mind, the $\mathrm{CN}$ nanosheet is convinced to be a semiconductor with wide indirect band gap.

The analysis of isosurface charge density could provide a comprehensive understanding of the orbital composition of electronic band. The highest valance state is comprised by atomic orbitals along the perpendicular direction, i.e., strong $\boldsymbol{\sigma}$ orbital between $\mathrm{C}$ atoms together with partial $p_{z}$ orbital of $\mathrm{N}$ atoms, and the second highest valance state is exclusively composed by partial $p_{z}$ orbitals perpendicular to atomic plane. For lowest and second lowest conduction band, both the in-plane and out-of-plane atomic orbitals take part in the band formation. Thus, the strong interlayer bonding substantially reshape the band structure and electronic properties of $\mathrm{CN}$ nanosheet.

We have plotted the variation of VBM and CBM obtained with PBE as functions of different tensile strains in Figs. 7(b)-(d). Generally, the band gap decreases with increasing tensile strain in the elastic region for tensile strain $\epsilon_{y y}$ and $\epsilon_{b i}$. For armchair tension $\epsilon_{x x}$, the band gap first decreases with increasing armchair tension, passing through a minimum, then increases and forms a direct band gap from $\epsilon_{x x}=0.12$ to $\epsilon_{x x}=0.14$. This direct band gap is a consequence of the intersection of CBM between $\Gamma$ and $\mathrm{M}^{\prime}$. With the increasing of these three tensions, there is tendency that the VBM at other high symmetry points ( $\mathrm{M}, \mathrm{K}$ and their splitting under uniaxial tension) are raised with regard to the VBM at $\Gamma$. However, the variations of CBM are much more complicated and shows strong anisotropy to the applied strain. Finally, variations of band gap with respect to the tensile strain are summarized in Fig. 7(e). It is notable that these band gap results are obtained at the PBE level and then corrected using the scissors operator method based on the $2.1 \mathrm{eV}$ difference between the PBE and PBE $+G_{0} W_{0}$ band gap at the $\Gamma$ point. Accordingly, the electronic band structure of $\mathrm{CN}$ nanosheet can be constructively controlled by tensile strain and the transition between direct and indirect band gaps can be realized. Similar trends can be also found for $2 \mathrm{D} X \mathrm{C}(X=\mathrm{Si}, \mathrm{Ge}, \mathrm{As})$ nanosheets in our early work [52]. Thus strained CN nanosheet have important and potential applications in optoelectronics and energy engineering.

\section{Conclusion}

In summary, we have investigated the elastic, vibrational and electronic properties of the $\mathrm{CN}$ nanosheet in the puckered honeycomb structure with covalent interlayer bonding using first-principles simulations. The strong interlayer $\boldsymbol{\sigma}$ bonding between $\mathrm{C}$ atoms together with the hybrid intralayer $s p^{3}$ bonding of $\mathrm{C}-\mathrm{N}$ atoms maintain the 
structure stability of $\mathrm{CN}$ nanosheet. It is found that $\mathrm{CN}$ nanosheet has an effective Young's modulus of $1013 \mathrm{GPa}$ which indicates comparable in-plane stiffness to monolayer graphene. A negative Poisson's ratio in the outof-plane direction under biaxial deformation, which is attributed to additional degree of atomic relaxation introduced by the interlayer bonding, is revealed. The highly anisotropic mechanical and electronic response of $\mathrm{CN}$ nanosheet to the tensile strain have been explored. It is identified that the tensile strength of $\mathrm{CN}$ nanosheets are dictated by the instability of out-of-plane transverse phonon branch ZA for armchair tension $\epsilon_{x x}$, but in-plane transverse phonon branch TA for the zigzag tension $\epsilon_{y y}$. Our results show that the band structure of $\mathrm{CN}$ nanosheet could be effectively tailored through strain engineering. This work demonstrates that $\mathrm{CN}$ nanosheet can be engineered through the controlling of covalent interlayer bonding and applied strain to enhance structure stability, increase buckling strength, and modify band gap, all indispensable characteristics for potential application in the nanoelectromechanical system.

Electronic supplementary material Electronic supplementary materials are available in the online version of this article at https:// doi.org/10.1007/s11467-021-1106-5 and http://journal.hep.com.cn/ fop/En/10.1007/s11467-021-1106-5 and are accessible for authorized users.

Acknowledgements This work was supported by the Special Program for Applied Research on Super Computation of the NSFC-Guangdong Joint Fund (the second phase) under Grant No. U1501501, and the Xiamen University Malaysia Research Fund, Grant No. XMUMRF/2019-C3/IORI/0001.

\section{References}

1. K. S. Novoselov, A. K. Geim, S. V. Morozov, D. Jiang, Y. Zhang, S. V. Dubonos, I. V. Grigorieva, and A. A. Firsov, Electric field effect in atomically thin carbon films, Science 306, 666 (2004)

2. Y. Zhang, Y. W. Tan, H. L. Stormer, and P. Kim, Experimental observation of the quantum Hall effect and Berry's phase in graphene, Nature 438, 201 (2005)

3. C. Berger, Z. Song, X. Li, X. Wu, N. Brown, C. Naud, D. Mayou, T. Li, J. Hass, A. N. Marchenkov, E. H. Conrad, P. N. First, and W. A. de Heer, Electronic confinement and coherence in patterned epitaxial graphene, Science 312 , 1191 (2006)

4. H. Nakano, T. Mitsuoka, M. Harada, K. Horibuchi, H. Nozaki, N. Takahashi, T. Nonaka, Y. Seno, and H. Nakamura, Soft synthesis of single-crystal silicon monolayer sheets, Angew. Chem. Int. Ed. 45, 6303 (2006)

5. S. Cahangirov, M. Topsakal, E. Aktürk, H. Şahin, and S. Ciraci, Two- and one-dimensional honeycomb structures of silicon and germanium, Phys. Rev. Lett. 102, 236804 (2009)
6. P. R. Wallace, The band theory of graphite, Phys. Rev. 71, 622 (1947)

7. H. Liu, A. T. Neal, Z. Zhu, Z. Luo, X. Xu, D. Tomanek, and P. D. Ye, Phosphorene: An unexplored 2D semiconductor with a high hole mobility, ACS Nano 8, 4033 (2014)

8. L. Li, Y. Yu, G. J. Ye, Q. Ge, X. Ou, H. Wu, D. Feng, $\mathrm{X}$. H. Chen, and Y. Zhang, Black phosphorus field-effect transistors, Nat. Nanotechnol. 9, 372 (2014)

9. J.-H. Lin, H. Zhang, and X.-L. Cheng, First-principle study on the optical response of phosphorene, Front. Phys. 10, 1 (2015)

10. J.-C. Zheng, H.-Q. Wang, A. Wee, and C. Huan, Structural and electronic properties of $\mathrm{Al}$ nanowires: An $a b$ initio pseudopotential study, Int. J. Nanosci. 1, 159 (2002)

11. Z.-Q. Wang, T.-Y. Lü, H.-Q. Wang, Y. P. Feng, and J.-C. Zheng, Review of borophene and its potential applications, Front. Phys. 14, 33403 (2019)

12. J.-C. Lei, X. Zhang, and Z. Zhou, Recent advances in MXene: Preparation, properties, and applications, Front. Phys. 10, 276 (2015)

13. C. Niu, Y. Z. Lu, and C. M. Lieber, Experimental realization of the covalent solid carbon nitride, Science 261, 334 (1993)

14. K. M. Yu, M. L. Cohen, E. E. Haller, W. L. Hansen, A. Y. Liu, and I. C. Wu, Observation of crystalline $\mathrm{C}_{3} \mathrm{~N}_{4}$, Phys. Rev. B 49, 5034 (1994)

15. H. W. Song, F. Z. Cui, X. M. He, W. Z. Li, and H. D. $\mathrm{Li}$, Carbon nitride films synthesized by $\mathrm{NH}_{3}$-ion-beamassisted deposition, J. Phys.: Condens. Matter 6, 6125 (1994)

16. A. Bousetta, M. Lu, A. Bensaoula, and A. Schultz, Formation of carbon nitride films on $\mathrm{Si}(100)$ substrates by electron cyclotron resonance plasma assisted vapor deposition, Appl. Phys. Lett. 65, 696 (1994)

17. Z. J. Zhang, S. Fan, and C. M. Lieber, Growth and composition of covalent carbon nitride solids, Appl. Phys. Lett. 66, 3582 (1995)

18. S. R. J. Pearce, P. W. May, R. K. Wild, K. R. Hallam, and P. J. Heard, Deposition and properties of amorphous carbon phosphide films, Diam. Relat. Mater. 11, 1041 (2002)

19. F. Claeyssens, G. M. Fuge, N. L. Allan, P. W. May, and M. N. R. Ashfold, Phosphorus carbides: Theory and experiment, Dalton Trans. 2004, 3085 (2004)

20. J. N. Hart, P. W. May, N. L. Allan, K. R. Hallam, F. Claeyssens, G. M. Fuge, M. Ruda, and P. J. Heard, Towards new binary compounds: Synthesis of amorphous phosphorus carbide by pulsed laser deposition, J. Solid State Chem. 198, 466 (2013)

21. A. Furlan, G. K. Gueorguiev, Z. Czigány, H. Högberg, S. Braun, S. Stafström, and L. Hultman, Synthesis of phosphorus-carbide thin films by magnetron sputtering, Phys. Status Solidi RRL 2, 191 (2008)

22. M. Côté and M. L. Cohen, Carbon nitride compounds with 1:1 stoichiometry, Phys. Rev. B 55, 5684 (1997)

23. J.-C. Zheng, M. C. Payne, Y. P. Feng, and A. T.-L. Lim, Stability and electronic properties of carbon phosphide compounds with 1:1 stoichiometry, Phys. Rev. B $67,153105(2003)$ 
24. G. Wang, R. Pandey, and S. P. Karna, Carbon phosphide monolayers with superior carrier mobility, Nanoscale 8, 8819 (2016)

25. A. K. Geim and I. V. Grigorieva, van der Waals heterostructures, Nature 499, 419 (2013)

26. K. S. Novoselov, A. Mishchenko, A. Carvalho, and A. H. Castro Neto, 2D materials and Van der Waals heterostructures, Science 353, aac9439 (2016)

27. X.-R. Hu, J.-M. Zheng, and Z.-Y. Ren, Strong interlayer coupling in phosphorene/graphene Van der Waals heterostructure: A first-principles investigation, Front. Phys. 13, 137302 (2017)

28. P. L. de Andres, R. Ramírez, and J. A. Vergés, Strong covalent bonding between two graphene layers, Phys. Rev. B 77, 045403 (2008)

29. J.-J. Li, Y. Dai, and J.-C. Zheng, Strain engineering of ion migration in $\mathrm{LiCoO}_{2}$, Front. Phys., doi:10.1007/s11467021-1086-5 (2021)

30. J. Kanasaki, E. Inami, K. Tanimura, H. Ohnishi, and K. Nasu, Formation of $s p^{3}$-bonded carbon nanostructures by femtosecond laser excitation of graphite, Phys. Rev. Lett. 102, 087402 (2009)

31. K. Nishioka and K. Nasu, Cooperative domain-type interlayer $s p^{3}$-bond formation in graphite, Phys. Rev. B 82, 035440 (2010)

32. S. Ghosh, W. Bao, D. L. Nika, S. Subrina, E. P. Pokatilov, C. N. Lau, and A. A. Balandin, Dimensional crossover of thermal transport in few-layer graphene, Nat. Mater. 9, 555 (2010)

33. Z. Wei, Z. Ni, K. Bi, M. Chen, and Y. Chen, In-plane lattice thermal conductivities of multilayer graphene films, Carbon 49, 2653 (2011)

34. T. Guo, Z.-D. Sha, X. Liu, G. Zhang, T. Guo, Q.-X. Pei, and Y.-W. Zhang, Tuning the thermal conductivity of multi-layer graphene with interlayer bonding and tensile strain, Appl. Phys. A 120, 1275 (2015)

35. P. Giannozzi, S. Baroni, N. Bonini, M. Calandra, R. Car, C. Cavazzoni, D. Ceresoli, G. L. Chiarotti, M. Cococcioni, I. Dabo, A. D. Corso, S. de Gironcoli, S. Fabris, G. Fratesi, R. Gebauer, U. Gerstmann, C. Gougoussis, A. Kokalj, M. Lazzeri, L. Martin-Samos, N. Marzari, F. Mauri, R. Mazzarello, S. Paolini, A. Pasquarello, L. Paulatto, C. Sbraccia, S. Scandolo, G. Sclauzero, A. P. Seitsonen, A. Smogunov, P. Umari, and R. M. Wentzcovitch, QUANTUM ESPRESSO: A modular and open-source software project for quantum simulations of materials, J. Phys.: Condens. Matter 21, 395502 (2009)

36. J. P. Perdew, K. Burke, and M. Ernzerhof, Generalized gradient approximation made simple, Phys. Rev. Lett. 77, 3865 (1996)

37. P. E. Blöchl, Projector augmented-wave method, Phys. Rev. B 50, 17953 (1994)
38. Z. H. Levine and D. C. Allan, Linear optical response in silicon and germanium including self-energy effects, Phys. Rev. Lett. 63, 1719 (1989)

39. V. Fiorentini and A. Baldereschi, Dielectric scaling of the self-energy scissor operator in semiconductors and insulators, Phys. Rev. B 51, 17196 (1995)

40. M. S. Hybertsen and S. G. Louie, Electron correlation in semiconductors and insulators: Band gaps and quasiparticle energies, Phys. Rev. B 34, 5390 (1986)

41. O. Zakharov, A. Rubio, X. Blase, M. L. Cohen, and S. G. Louie, Quasiparticle band structures of six II-VI compounds: ZnS, ZnSe, ZnTe, CdS, CdSe, and CdTe, Phys. Rev. B 50, 10780 (1994)

42. P. E. Trevisanutto, C. Giorgetti, L. Reining, M. Ladisa, and V. Olevano, $A b$ initio $G W$ many-body effects in graphene, Phys. Rev. Lett. 101, 226405 (2008)

43. H. Şahin, S. Cahangirov, M. Topsakal, E. Bekaroglu, E. Akturk, R. T. Senger, and S. Ciraci, Monolayer honeycomb structures of group-IV elements and III-V binary compounds: First-principles calculations, Phys. Rev. B 80, $155453(2009)$

44. C. Lee, X. Wei, J. W. Kysar, and J. Hone, Measurement of the elastic properties and intrinsic strength of monolayer graphene, Science 321, 385 (2008)

45. J.-W. Jiang, Graphene versus $\mathrm{MoS}_{2}$ : A short review, Front. Phys. 10, 287 (2015)

46. F. Liu, P. Ming, and J. Li, Ab initio calculation of ideal strength and phonon instability of graphene under tension, Phys. Rev. B 76, 064120 (2007)

47. J. Wu, B. Wang, Y. Wei, R. Yang, and M. Dresselhaus, Mechanics and mechanically tunable band gap in singlelayer hexagonal boron-nitride, Mater. Res. Lett. 1, 200 (2013)

48. J.-W. Jiang, T. Chang, X. Guo, and H. S. Park, Intrinsic negative Poisson's ratio for single-layer graphene, Nano Lett. 16, 5286 (2016)

49. G. Qin and Z. Qin, Negative Poisson's ratio in twodimensional honeycomb structures, npj Comput. Mater. 6, 51 (2020)

50. T. Li, J. W. Morris, N. Nagasako, S. Kuramoto, and D. C. Chrzan, "Ideal" engineering alloys, Phys. Rev. Lett. 98, 105503 (2007)

51. T. Li, Ideal strength and phonon instability in single-layer $\mathrm{MoS}_{2}$, Phys. Rev. B 85, 235407 (2012)

52. T.-Y. Lü, X.-X. Liao, H.-Q. Wang, and J.-C. Zheng, Tuning the indirect-direct band gap transition of $\mathrm{SiC}, \mathrm{GeC}$ and $\mathrm{SnC}$ monolayer in a graphene-like honeycomb structure by strain engineering: A quasiparticle $G W$ study, $J$. Mater. Chem. 22, 10062 (2012) 


\section{RESEARCH ARTICLE}

\section{Ab initio study of anisotropic mechanical and electronic properties of strained carbon-nitride nanosheet with interlayer bonding}

Hao Cheng ${ }^{1,2}$, Jin-Cheng Zheng ${ }^{1,2, \dagger}$

${ }^{I}$ Department of Physics and the Collaborative Innovation Center for Optoelectronic Semiconductors and Efficient Devices, Xiamen University, Xiamen 361005, China

${ }^{2}$ Department of Physics, Xiamen University Malaysia, 439000, Sepang, Selangor, Malaysia

Corresponding author.E-mail: ${ }^{\dagger} j c z h e n g @ x m u . e d u . c n$

\section{SUPPLEMENTARY MATERIAL}

Table S1 The calculated structure parameters of CN nanosheet in LB (low-buckled) geometry without interlayer bonding and planar (PL) geometry. The values of angle between intralayer bond C-N-C $(\alpha)$, C-N bond length $\left(d_{\mathrm{CN}}\right)$, lattice constant $(a)$, and cohesive energy $\left(E_{\mathrm{coh}}\right)$ are given.

\begin{tabular}{ccccc}
\hline \hline Structure & $\alpha$ & $d_{\mathrm{CN}}$ & $a$ & $E_{c o h}$ \\
& $\left({ }^{\circ}\right)$ & $(\AA)$ & $(\AA)$ & $(\mathrm{eV} /$ atom $)$ \\
\hline LB geometry without interlayer bonding & 119.1 & 1.390 & 2.396 & 7.994 \\
Planar geometry & 120.0 & 1.385 & 2.399 & 7.992 \\
\hline \hline
\end{tabular}

(a)

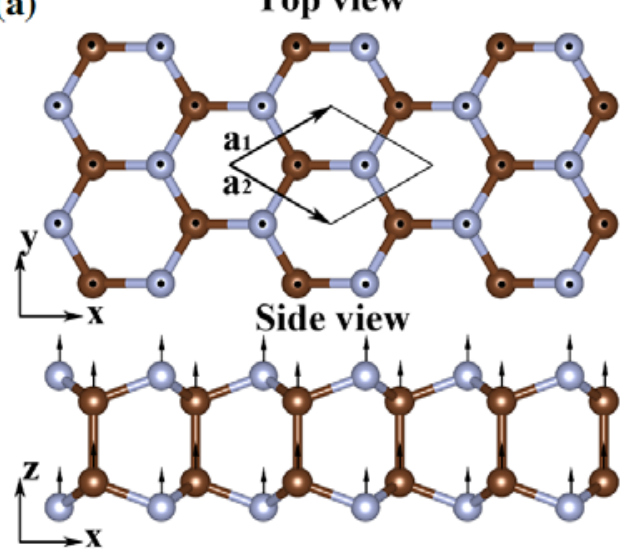

(b)

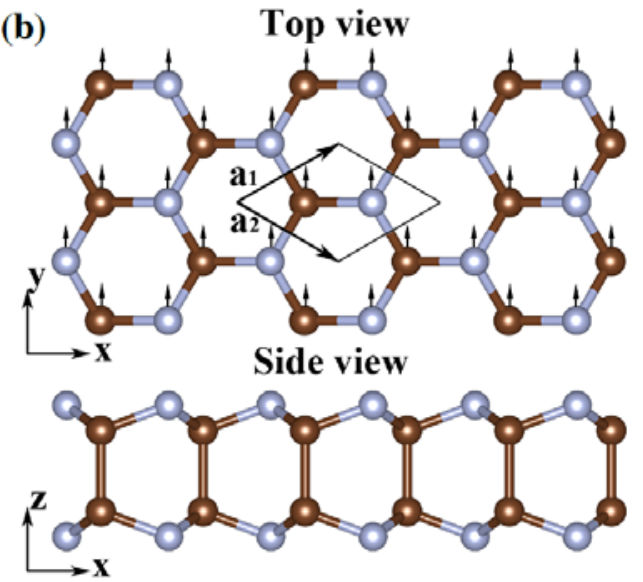

Fig. S1 The illustration of unstable eigenvector corresponding to (a) out-of-plane transverse phonon branch ZA at $\varepsilon_{x x}=0.13$ and (b) in-plane transverse phonon branch TA at $\varepsilon_{y y}=0.18$. 

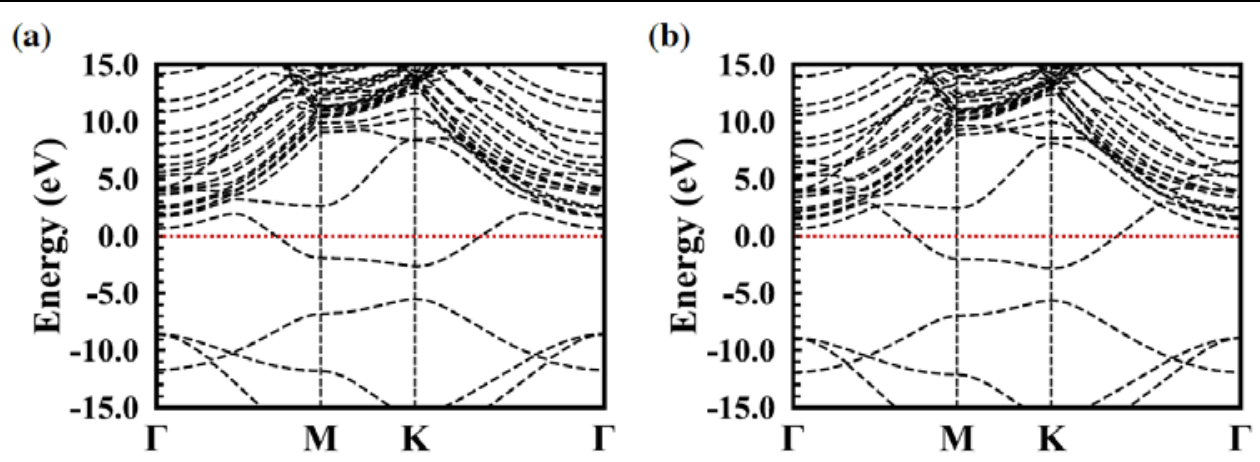

Fig. S2 Calculated electronic band structures of CN nanosheet in (a) LB geometry without interlayer bonding and (b) planar (PL) geometry are presented. The Fermi energy is set to be zero. 